\title{
Southeast Asia is an emerging hotspot for COVID-19
}

To the Editor-The rise in COVID-19 cases in India has become an important lesson to the global community ${ }^{1}$. Because certain parts of the world have been left vulnerable to COVID-19, a humanitarian crisis at an unprecedented scale has emerged and spread to the rest of the world. The SARS-CoV-2 Delta variant (B.1.617.2) that evolved from the outbreak in India has set back the success of COVID-19 control measures in several developed countries ${ }^{2}$. Southeast Asia and its population of more than 655 million people could be the next hotspot due to geographical and socioeconomic factors, such as ongoing political unrest and the emergence of new variants.

Southeast Asia is now being bombarded with three known variants of concern:

B.1.1.7 (Alpha), B.1.351 (Beta) and B.1.617.2 (Delta). The movement of workers between countries, often through illegal and unmonitored channels, in combination with a limited vaccine rollout, has put the whole region at risk from highly transmissible and immunoevasive variants. However, little information about this rising crisis is seen in the media or from the global medical community.

In 2020, Southeast Asia was the role model in terms of COVID-19 control. The situation in mid-2021 has been transformed by the rise in case numbers. The average number of confirmed daily new cases in Southeast Asia has increased from more than 12,900 in March 2021 to 28,800 in June 2021, with a median time-varying reproduction number of 1.09 , which indicates that the daily new cases are growing exponentially (Fig. 1a). The data are probably under-reported due to limited testing capacity.

The degree of genetic variation in the available SARS-CoV-2 genomes in Thailand suggests that the virus has been propagating without detection for months ${ }^{3}$. From March 2021 to June 2021, the spread of the Alpha and Delta variants took over the region, which resulted in a collapse of the healthcare system. Starting in March 2021, the Alpha variant was circulating in Cambodia and Thailand (Fig. 1b). The public-health measures successfully implemented during the first wave in 2020 failed to control the new variant. The positivity rate of SARS-CoV-2 tests was as much as $60-90 \%$ in certain clusters in Thailand. Cases of COVID-19 have spilled into Laos, which

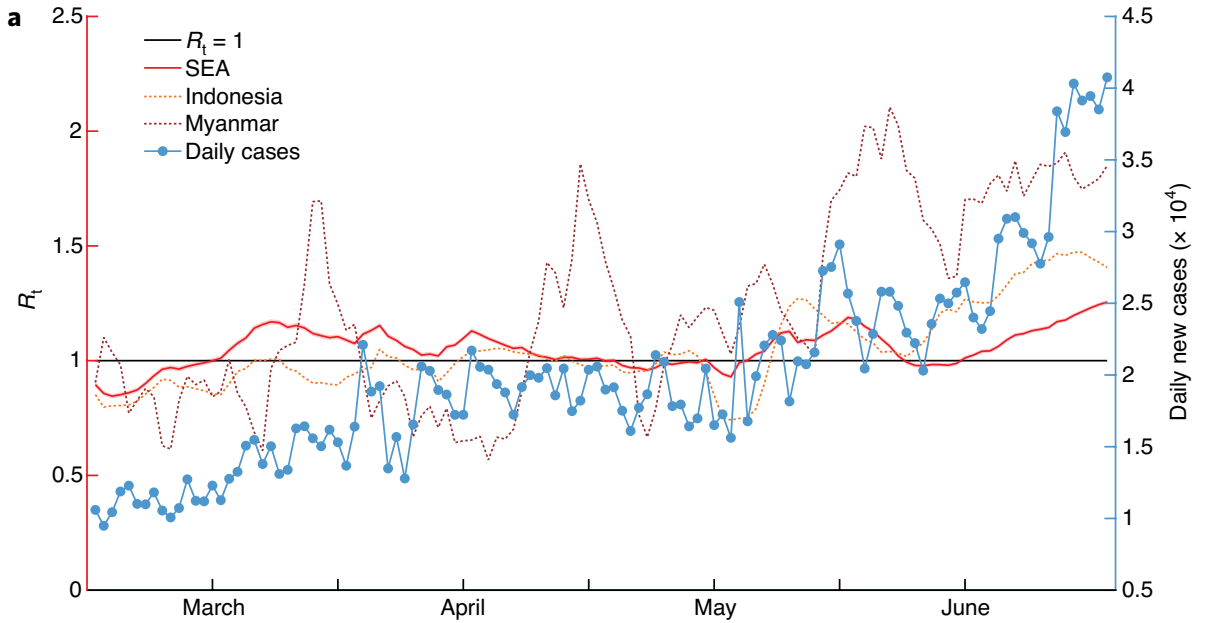

b

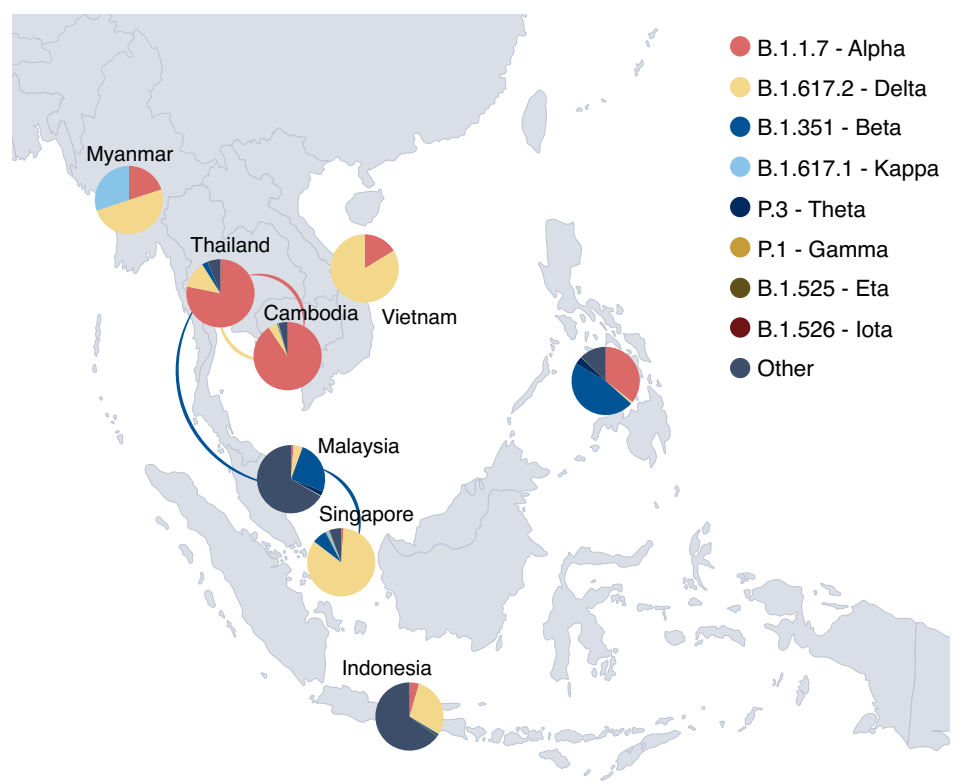

Fig. 1 | Overview of the COVID-19 situation in Southeast Asia. a, Daily case number and time-varying reproduction number $\left(R_{\mathrm{t}}\right)$ during the period of 1 March 2021 to 30 June 2021 (ref. ${ }^{5}$ ). Plot shows reproduction numbers from Southeast Asia (SEA), Indonesia (the country with the highest case number) and Myanmar. On the basis of existing data from Myanmar, the reproduction number has already exceeded 1.0. b, Proportion of SARS-CoV-2 variants during the period of March 2021 to June 2021, presented as pie charts with colors according to Pango lineages (https://cov-lineages.org/index.html; database version 2021-06-15) and World Health Organization classification. Lines linking the pie charts indicate putative connections of viral populations (same Pango color code) between countries. The regional analysis was based on the sequencing data from COVID-19 Network Investigations and from other countries in Southeast Asia deposited at the GISAID database (https://www.epicov. org/epi3/; database version 2021-06-28) with genomes associated with Cambodia (300), Indonesia (831), Malaysia (486), Myanmar (10), Philippines (1,290), Singapore (769), Thailand (872) and Vietnam (86). Detailed data visualization is available in the Nextstrain format at https://coni.team/resource/. Publ. note: Springer Nature is neutral about jurisdictional claims in maps. 
used to have almost no local transmission. Indonesia and Malaysia have been hit with the Beta variant, which is known to reduce vaccine effectiveness ${ }^{4}$. The virus has already spread to the southern part of Thailand.

Since April 2021, Southeast Asia has been plagued by competition between Alpha and Delta variants, with the percentage of the Delta variant rising in Indonesia, Malaysia, Thailand and Vietnam. On the basis of available genome sequences, the Delta variant seems to have successfully overtaken the Alpha variant in Indonesia and Vietnam. Most importantly, the genomic data have confirmed uncontrolled movements of these variants between countries, and with limited vaccine rollout (as of 30 June 2021, less than $5 \%$ of the total population was fully vaccinated), Southeast Asia as a whole is becoming a new regional hotspot in the global fight against COVID-19.

The biggest under-reported crisis is likely to be in Myanmar. After the political unrest that started in February 2021, case reports and genomic surveillance activities became scarce. COVID-19 outbreaks with the Alpha variant are ongoing in Myanmar, on the basis of genomic data from imported cases in Singapore and Thailand. Myanmar is exposed to the Alpha, Beta and Delta variants both from Thailand in the East and from Bangladesh and India in the West, and therefore it might not be able to avoid the importation of these variants, especially given that human populations are moving in and out of the country because of political instability.

While the high-income countries of the world are enjoying the return of a pre-pandemic lifestyle, new vaccine-escape variants in Southeast Asia could set back the global success of COVID-19 control in its final mile. An urgent increase in vaccine supplies must be distributed to local hotspots in Southeast Asia, guided by viral surveillance data, in order to put an end to this devastating pandemic.

Thanat Chookajorn (D) 1,2,3凶,
Theerarat Kochakarn (D) 1,3,
Chaiwat Wilasang 4,5, Namfon Kotanan ${ }^{1,2}$ and
Charin Modchang,
${ }^{1}$ COVID-19 Network Investigations Alliance,
Bangkok, Thailand. ${ }^{2}$ Genomics and Evolutionary
Medicine Unit, Center of Excellence in Malaria
Research, Faculty of Tropical Medicine, Mahidol
University, Bangkok, Thailand. ${ }^{3}$ Molecular Infection
Medicine Sweden, Molecular Biology Department,
Umeå University, Umeå, Sweden. ${ }^{4}$ Biophysics Group,
Department of Physics, Faculty of Science, Mahidol

University, Bangkok, Thailand. ${ }^{5}$ Thailand Center of Excellence in Physics, Commission on Higher Education, Bangkok, Thailand.

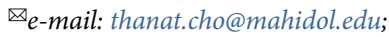
charin.mod@mahidol.edu

Published online: 16 August 2021 https://doi.org/10.1038/s41591-021-01471-x

References

1. Singh, J., Rahman, S. A., Ehtesham, N. Z., Hira, S. \& Hasnain, S. E. Nat. Med. 27, 1131-1133 (2021).

2. Campbell, F. et al. Euro Surveill. 26, 2100509 (2021).

3. Joonlasak, K. et al. Virus Res. 292, 198233 (2020).

4. Abu-Raddad, L. J., Chemaitelly, H. \& Butt, A. A. N. Engl. J. Med. 385, 187-189 (2021).

5. Wilasang, C. et al. J. Travel Med. 27, taaa095 (2020).

Acknowledgements

We thank E. Batty for critical reading of the manuscript and discussion. The COVID-19 genomic surveillance program at COVID-19 Network Investigations Alliance was supported by the National Research Council of Thailand, Thailand Center of Excellence for Life Sciences, Mahidol University, Ramathibodi Foundation and the Wellcome Trust. C.M. was supported by the National Research Council of Thailand and the Thailand Center of Excellence in Physics.

Author contributions

T.C., T.K. and C.M. wrote the manuscript, and all authors analyzed the data and edited the manuscript.

Competing interests

The authors declare no competing interests. 